\title{
The Safety and Efficacy of SGLT2 Inhibitors in Diabetes Mellitus Management
}

\author{
Dominic Adam Worku* \\ University Hospital of Wales, Cardiff, UK
}

*Corresponding Author: Dominic Adam Worku, University Hospital of Wales, Cardiff, UK Email: dominicworku@hotmail.co.uk

\begin{abstract}
Diabetes is a global health challenge which requires better approaches towards its management. Currently, physicians must employ several strategies to help control the hyperglycaemia and comorbid disease which features as part of diabetes and the metabolic syndrome. Sodium Glucose Co-transport 2 Inhibitors (SGLT2i) are a new class of anti-diabetic medications which have several important properties including a low risk of hypoglycaemia and renal and cardio protective effects which make them attractive in the long-term management of diabetes. However ongoing issues exist in how best they should be implemented in patients who are often on other anti-diabetic medication and which patients would best benefit from them. This mini- review will consider these issues.
\end{abstract}

Keywords: Diabetes Mellitus, SGLT2 inhibitors, Management, Cardiac Failure, Diabetic Kidney Disease

\section{INTRODUCTION}

Diabetes Mellitus (DM) is a global health problem which as of 2014 was estimated to affect 422 million adults with its prevalence in low and middle income countries set to rise (1.)While this condition has plagued humanity since the dawn of civilization it was only in 1936 that it became apparent that there were two main types aptly named Type 1 and Type 2 DM which while similar often require different treatment regimens and goals. While the former of these has an autoimmune basis the latter is the result of impaired insulin secretion and sensitivity in the peripheral tissues and the linked metabolic syndrome. This describes several conditions including obesity, dyslipidaemia and hyperglycaemia/insulin resistance which predispose individuals to T2DM and Cardiovascular disease (CVD) (2). It is of no surprise therefore that risk factors for T2DM development include physical inactivity, sedentary lifestyle, smoking, alcohol excess and obesity with the role of genetics increasingly becoming apparent in T2DM pathogenesis.

The management of type 2 diabetic patients involves optimisations of glycated haemoglobin levels $(\mathrm{HbA} 1 \mathrm{c})$. This biomarker is very important in stratifying patients according their risk of micro/macro vascular complications, with $\mathrm{HbA1c}<7 \%$ significantly reducing the likelihood of complications. As such it is imperative given the rise in T2DM cases to tightly control blood glucose and $\mathrm{HbA1c}$ concentrations while limiting any potential hypoglycaemic episodes (3).

In order to achieve glycaemic control multiple methods are employed by clinicians including lifestyle modification, pharmacological intervention and even surgery.

Indeed in healthy individual's glucose homeostasis is complex process which involves interplay between glucose production, reabsorption and utilization with the kidney and liver both central to this. However, current pharmacological therapies are plagued with undesirable side effects including hypoglycaemia, GI symptoms, weight gain and renal toxicity (4).

Sodium-Glucose Co-transporter 2 inhibitors (SGLT2i) are a new and exciting class of antidiabetic medications which work by inhibiting kidney tubular glucose reabsorption through Sodium Glucose Transport Proteins (SGLTs) binding. These membranous glucose co transporters are found on the brush border of the proximal tubule and are responsible for $80-90 \%$ of tubular glucose reabsorption. In contrast to 
SGLT1 transporters SGLT2 transporters have high capacity but low affinity for glucose (5). Indeed due to the hyperglycaemia of DM, SGLT2 transporter genes are up regulated in patients with T2DM and as such leads to a perpetual worsening of the patient's hyperglycaemia. SGLT2i inhibitors therefore work to promote the urinary excretion of glucose however their use has been theorised to promote increased hepatic glucose production through the up regulation of counter-regulatory hormones (6). However as this particular class of drug is insulin independent in its mechanism of action it minimizes any risk of hypoglycaemia and can therefore be used at any stage of T2DM management but does highly rely on underlying kidney function which can be severely compromised in this setting (7).

\section{MATERiAl AND MethodS}

Clinical studies were identified using the PUBMED and COCHRANE databases. All papers were published in peer reviewed journals in the last 10 years. Papers were selected if they attempted to explore the use of SGLT2i therapy in Diabetes management and associated comorbid disease and/or their safety profile in the setting of comorbid disease.

\section{RESULTS AND DISCUSSION}

The first SGLT2i, Phlorizin, a type of flavinoid was isolated from the bark of the apple tree in 1835, although it took until the 1970s to understand its mechanism of action (4). Early trials of this compound in diabetic rats showed it was able to normalize fasting and postprandial glucose levels with paralleled improvements in both insulin sensitivity and responsiveness. However, this compound has non-specific SGLT1 and SGLT2 activity the former of these being located in the gastrointestinal tract causing several poorly tolerated side effects. Indeed when used in humans Phlorizin exhibited limited efficacy due to poor bioavailability (5).

Due to these promising results scientist searched for effective related compounds. One of the first approved SGLT2i was Canagliflozin with Dapagliflozin and Empagloflizin quickly following (3). All three of these compounds are approved within Europe. Canagliflozin has been shown to be able to reduce the increased renal threshold for glucose reabsorption in diabetics from $415 \mathrm{mg} / \mathrm{dL}$ to $94 \mathrm{mg} / \mathrm{dL}$ with dapagliflozin demonstrating significant $\mathrm{HbA1c}$ reductions; fasting and postprandial glucose levels and glucosuria mediated related calorie loss of between $200-300 \mathrm{kcal} / \mathrm{day}$. In a meta-analysis of studies regarding currently marketed SGLT2i a mean reduction in $\mathrm{HbA} 1 \mathrm{c}$ levels of $0.77 \%$ (0.5$1 \%$ ) which was dose dependent was recorded. Importantly however did not necessarily correlate with total body weight loss but did with pre-therapy HbA1c levels (8).

Due to this glucosuria, patients can expect to lose between $1.5-3.5 \mathrm{~kg}$ within $\sim 3-4$ months of drug initiation and is predominantly from body fat as illustrated by Dual Energy X-ray absorptiometry (DEXA) scanning. Such weight reductions are importantly maintained over the long term with dapagliflozin $10 \mathrm{mg}$ maintain this weight loss for up to 102 weeks after drug initiation(6) (8).

In addition SGLT2i therapy provide improvements in blood pressure secondary to osmotic diuresis of $\sim 5 \mathrm{mmHg}$ systolic blood pressure and $0.9 \mathrm{mmHg}$ in $24 \mathrm{~h}$ ambulatory mean systolic BP (9). This once again helps alleviate the CVD risk that these patients are at risk of. Interesting new effects though are being seen through their routine use with empagliflozin demonstrating the ability to increase $\beta$-cell function and lipid profile in diabetics while exhibiting renoprotective propertiesby helping to reduce the hyperfiltration mechanism and expression of key molecules including hypoxia inducible factor 1(HIF1) and NF-kB which are key mediators of diabetic kidney disease(6).

It is as a result of this combination of properties including $\mathrm{HbA} 1 \mathrm{c}$ lowering effects, reductions in body weight, improvements in blood pressure and little risk of hypoglycaemia that makes these agents so attractive in diabetes management with an overall $29 \%$ decreased risk of death from all causes (10).

Heart Failure is a comorbid condition that frequently affects individuals with DM. In diabetic patients complicated by heart failure the optimal management of the hyperglycaemia remains a challenge. This is because some of the current pharmacological therapies in particular thiazolidinediones and DPP-4 inhibitors have been linked to increased risk of heart failure. Of the SGLT2 inhibitors, empagliflozin has been uniquely shown to reduce cardiovascular risk and mortality within 1 month after the initiation of therapy (37\%- relative risk 0.63 [0.51-0.77]) and were significantly less likely to require loop diuretics in their heart failure management. The mechanism of this decrease is thought to include 
osmotic diuresis leading to decreased blood pressure, decreased vascular resistance, increased haematocrit and haemoglobin and increases in anti-oxidant levels as oxidative stress leads to cardiac remodelling and decreased cardiac contractility. Additionally, it is thought that as a result of SGLT2i use the heart becomes more efficient as it switches from free fatty acid to ketone metabolism (11).

However one issue is the use of SGLT2i as a routine adjunct to other anti-diabetic agents including insulin. Currentli the most common adjunct used in T1DM management currently is metformin of the biguanide class, however while its use is linked with significant reductions in weight and $\mathrm{HbAlC}$ in the short term they are not maintained over longer time periods with other drug classes including thiazodiladiones, GLP1, DPP-4 inhibitors remaining unstudied in this respect. Complicating this matter further is the fact that there is a paucity of studies looking at SGLT2 inhibitor use in T1DM and they often utilise a small number of patients during a limited study period. As such the conclusions made from them are of limited use; as such therapy must be used for life (12). That being said it has been demonstrated that SGLT2 inhibitor use within T1DM can lead to decreased insulin requirements of up to $16 \%$, this is particularly of benefit in the young who often omit insulin for fear of its anabolic effects (7). In addition in T1DM it was found that use of compounds such as dapagliflozin could increase glucosuriaby $88 \mathrm{mg} /$ day and a recent meta-analysis found that the improvements SGLT2i could have on DM management when used as an add on therapy to insulin were dose independent with body weight reductions increased in T2DM patients versus T1DM and improved glucose control in particularly eastern Asian patients (13).

One of the main problems however with SGLT2i use in this arena is that it may cause euglycaemic diabetic ketoacidosis (DKA) in both T1DM and T2DM when used in in combination with insulin(12). The method by which this occurs is suggested that as SGLT2 $\mathrm{i}$ reduce blood glucose levels they reduce insulin secretion which in turns leads to a reciprocal increase in free fatty acid concentration and therefore ketone production in the liver. In addition SGLT2i's have been found within alpha cells on the pancreas and as such have been found to increase glucagon production which can mediate carnitine palmitoyl transferase-I activity which is a potent inducer of free fatty acid oxidation further increasing ketone production(14). Indeed there are many case reports of this with other atypical side effects including cerebral infarction in the elderly and as such it is considered SGLT2i therapy should be reserved for young obese patients. The euglyaemia described is thought to be as a result of both insulin deficiency and resistance which are milder in this setting resulting in moderate glucose levels with ketosis. While this side effect may happen at any time, with most reports reporting euglycaemic DKA occur within 1-14 days of initiation of therapy but have been documented up to several months later(16).It is a result of this that it is being encouraged that during sick days patients should discontinue SGLT2i therapy .

Like any medication, both clinicians and patients must reach an accord on the ability of a drug to deliver a positive benefit: risk ratio. In truth the incidence of adverse events is estimated between $57-83 \%$ which is similar to other accepted diabetic therapies (16). These may include increased risk of hypoglycaemia although this is usually mild to moderate in severity and the risk of urinary tract or genital infection. The latter of these represent the most frequent side effect of SGLT2i therapy (5\% incidence) and are a direct result of the glucosuric effect of SGLT2i. As such women and men should be taught the benefits of good perineal hygiene and may even benefit from prophylactic therapy (7)(13). Importantly, while SGLT2i therapy predicates on diuresis to remove the additional glucose load the frequency of hypotensive episodes occur in only $1.2-1.3 \%$ of patients with no significant impact noted on the rate ofvenous thromboembolism events (10)

Of some concern, observational studies have highlighted a trend to increased incidence of particularly bladder cancer in the long-term use of dapagliflozin (17). However this on further study has been found to not be significant as dapagliflozin has not been found to affect bladder cancer cell growth or survival. Moreover, it has been found that SGLT1 is over expressed in many cancer types and its inhibition has been found to sensitize prostatic cancer cells to monoclonal and Tyrosine Kinase based therapy and in pancreatic adenocarcinoma significantly correlates with disease free survival (17). 
As mentioned earlier as this drug works on the kidney its use within Chronic Kidney Disease (CKD) patients is of importance considering that DM predisposes to it. Studies using dapagliflozin have shown that when used in T2DM patients with Stage $3 \mathrm{CKD}$, the glucosuric effect is diminished by $\sim 50 \%$ and yield insignificant decreases in both $\mathrm{HbA1c}$ levels and fasting blood glucose but did not increase the risk of acute renal toxicity. This however is not the case with canagliflozin at doses of $100 \mathrm{mg}$ and $300 \mathrm{mg}$, which remains effective in its anti-diabetic effect while causing a reversible decline in eGFR and an increase in albuminuria but no increased risk of kidney disease or acidosis (10). Indeed the EMPA-REG OUTCOME trial showed that the routine use of empagliflozinin T2DM patients resulted in a reduction in the incidence or worsening of nephropathy and risk of death of CVD by $~ 40 \%$ and similar reductions in the requirement for the initiation of renal replacement therapy(16).As such SGLT2 inhibitor use within CKD patients remains a topic of debate (5)(6) .

The big questions is which patients and when would benefit from this therapy. Current opinion is that SGLT2I are effective at any stage of T2DM as add on therapy or as a monotherapy in patients who are intolerant to metformin. However as mentioned having good baseline renal function remains important, but in general younger patients with established cardiovascular disease and are overweight are likely the type of patient whom will benefit most from this therapy (15). Upon starting SGLT2i it is recommended that lower doses be used and titrated while monitoring other diabetic medications. As mentioned this may include decreasing the patients insulin dose by $\sim 20 \%$ with sulphonylureas reduced or stopped. With regards to other therapist both DPP-4, GLP-1 and metformin the dose should be reviewed for gastrointestinal side effects however as this therapy has been found to be superior to either of these drug classes they too may be omitted from the patients medications (16)(18).

\section{Conclusion}

SGLT2i are a novel therapeutic class which could revolutionise diabetic management. While much research has highlighted the improvements in metabolic, cardiovascular and renal parameters that they afford patients there are ongoing issues which require further investigation. This is because SGLT2 inhibitors only reduce $30-50 \%$ glucose reabsorption from the kidney and this imbalance remains poorly understood. Additionally, greater understanding is required regarding the nature of complications that long-termuse of these agents affords especially when alongside traditional antidiabetic medications. This can only be achieved by more robust clinical trials with larger cohorts. However, it seems that this class of drug will in the years to come likely curtail the likelihood of complications in the surge in patients soon to be diagnosed with this chronic and life changing disease.

\section{REFERENCES}

[1] Global Report on Diabetes. World Health Organization 2016. https://www.google. co.uk/ url sa $=\mathrm{t} \& \mathrm{rct}=\mathrm{j} \& \mathrm{q}=\& \mathrm{esrc}=\mathrm{s} \&$ source $=$ web $\& \mathrm{~cd}=$ $2 \&$ ved $=0$ ahUKEwip5ZuDxKrXAhXSEOwKH brYAbEQFggwMAE\&url=http\%3A\%2F\%2Fa pps.who.int $\% 2$ Firis $\% 2$ Fbitstream $\% 2$ F $10665 \% 2$ F204871\%2F1\%2F9789241565257_eng.pdf\&u $\mathrm{sg}=\mathrm{AOvVaw} 27 \mathrm{PRH} 2 \mathrm{w} 5 \mathrm{js}$ ITVu-ELGGE74 (Accessed 6 $^{\text {th }}$ November 2017).

[2] Huang PL. A comprehensive definition for metabolic syndrome. Dis Model Mech.2009; 5: 231-237.

[3] Chen M, Xie CG, Gao H, Zheng H, Chen Q, Fang JQ. Comparative effectiveness of sodiumglucose co-transporter 2 inhibitors for controlling hyperglycaemia in patients with type 2 diabetes: protocol for a systematic review and network meta-analysis. BMJ.2016: http://bmjopen.bmj.com/content/6/1/e010252 (Accessed 1st November 2017).

[4] Choi CI. Sodium-Glucose Contransporter 2 (SGLT2) Inhibitors from Natural Products: Discovery of Next-Generation Antihyper glycemic Agents. Molecules.2016; 21(9).

[5] Andrianesis V, Glykofridi S, Duops J. The renal effects of SGLT2 inhibitors and a minireview of the literature. TherAdvEndocrinol Metao.2016; 7(6):212-228.

[6] Zou H, Zhou B, Xu G. SGLT2 inhibitors: a novel choice for the combination therapy in diabetic kidney disease. Cardiovascular Diabetology.2017. https://cardiab.Biomed central.com/articles/10.1186/s12933-017-05471 (Accessed $3{ }^{\text {rd }}$ Novemeber 2017)

[7] Kaira S. Sodium-Glucose Co-Transporter-2 (SGLT2) inhibitors: A review of their basic and clinical pharmacology. Diabetes Ther.2014; 5(2):255-366.

[8] Nuack MA. Update on developments with SGLT2 inhibitors in the management of type 2 diabetes. Drug Des Devel Ther.2014; 8:13351380. 
[9] O Steen et al. The role of sodium-glucose cotransporter 2 inhibitors in the management of type 2 diabetes. Can $J$ Diabetes. 2017; 41(6):517-523.

[10] Hackethal V. Cardiovascular Protection from SGLT2 inhibitors. Endocrinology Network.2016:http://www.endocrinologynetwo rk.com/sglt2/cardiovascular-protection-sglt2inhibitors (Accessed 1st November 2017)

[11] Pham D, R Albuquerque, McGuire DK, England IJ. Impact of empagliflozin in patients with diabetes and heart failure. Trends Cardiovasc Med.2017; 27(2):144-151.

[12] Chen J, Fan F, Wang JY, Long Y, Gao CL, Stanton RC, Xu Y. The efficacy and safety of SGLT2 inhibitors for adjunctive treatment of type 1 diabetes: a systematic review and metaanalysis. Scientific Reports.2017. https://www .nature.com/articles/srep44128?WT.feed_name =subjects_metabolic-diseases\&error $=$ cookies not_supported\#results (Accessed $31^{\text {st }}$ October 2017).

[13] Yang Y et al. Safety and efficiency of SGLT2 inhibitor combining with insulin in subjects with diabetes. Medicine (Baltimore). 2017; 96(21).
[14] Ogawa W, Sakaquchi K. Euglycemic diabetic ketoacidosis induced by SGLT2 inhibitors: a possible mechanism and contributing factors. $J$ Diabetes Investig. 2016; 7(2):135-138.

[15] Miyauchi M, Toyoda M, Fukagawa M. Atypical Ketoacidosis and Protracted Hyperglycosuria after Treatment with Ipragliflozin an SGLT2 inhibitor. InternMed. 2017; 56(13):1673-1678.

[16] Gomez-Peralata F et al. Practical approach to initiating SGLT2 inhibitors in type 2 diabetes. Diabetes Ther.2017; 8(5):953-962.

[17] Lin HW, Tseng $\mathrm{CH}$. A review on the relationship between SGLT2 inhibitors and cancer. Int $J$ Endocrinol.2014. https://www. ncbi.nlm.nih.gov/pmc/articles/PMC4164126/ (Accessed 30 ${ }^{\text {th }}$ November 2017).

[18] Handelsman $\mathrm{Y}$ et al. American association of clinical endocrinologists and american college of endocrinology position statement on the association of SGLT2 inhibitors and diabetic ketoacidosis. Endocr Pract. 2016; 22(6): 753762.

Citation: Dominic Adam Worku. The Safety and Efficacy of SGLT2 Inhibitors in Diabetes Mellitus Management. ARC Journal of Diabetes and Endocrinology. 2017; 3(1):19-23. doi:dx.doi.org/10.20431/24555983.0301003.

Copyright: (c) 2017 Authors. This is an open-access article distributed under the terms of the Creative Commons Attribution License, which permits unrestricted use, distribution, and reproduction in any medium, provided the original author and source are credited. 